The American Journal of Chinese Medicine, Vol. 43, No. 2, 383

(C) 2015 World Scientific Publishing Company

Institute for Advanced Research in Asian Science and Medicine

DOI: $10.1142 / S 0192415 X 15920019$

\title{
ERRATUM \\ Effect of the Semen Extract of \\ Thuja orientalis on Inflammatory Responses in Transient Focal Cerebral Ischemia Rat Model and LPS-Stimulated BV-2 Microglia
}

[The American Journal of Chinese Medicine, 41(1): 99-117, 2013]

Hyo Won Jung, ${ }^{*}$ Seok Yong Kang, ${ }^{\dagger}$ Ki Ho Park, ${ }^{\dagger}$ Tae Woo Oh, ${ }^{\dagger}$ Jin Ki Jung, ${ }^{\dagger}$

Song Hee Kim, ${ }^{*}$ Dong-Jun Choi ${ }^{*}$ and Yong-Ki Park ${ }^{*}{ }^{*}$

*Oriental Medicine R\&D Center, Dongguk University

Gyeongju 780-714, Republic of Korea

Department of Herbology, College of Oriental Medicine

Dongguk University, Gyeongju 780-714, Republic of Korea

Department of Oriental Internal Medicine

Dongguk University Ilsan Oriental Hospital

Gyeonggido 410-773, Republic of Korea

Published 26 March 2015

The left Figure $4(C)$ is corrected to the right one:

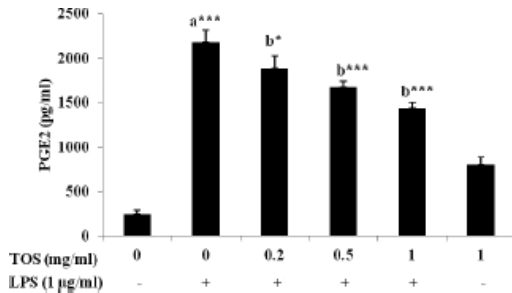

(A)

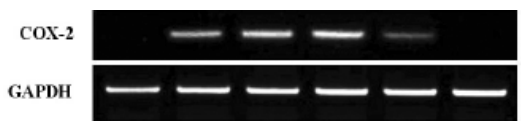

(B)

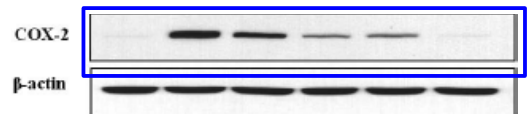

(C)

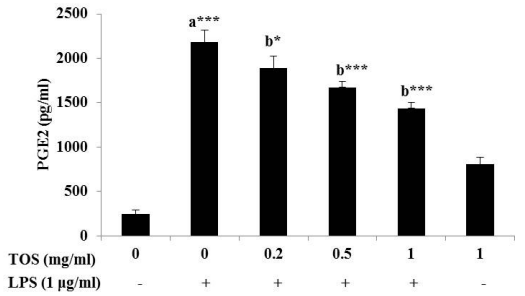

(A)

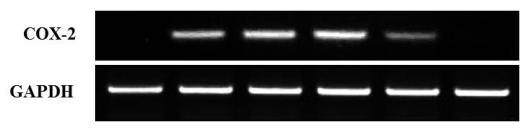

(B)
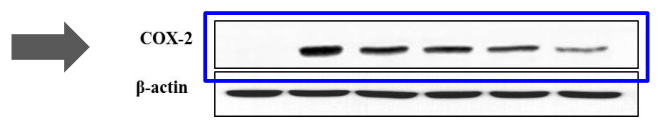

(C) 